\title{
Stress Relaxation in High Density Polyethylene. Effects of Orientation and Gamma Radiation
}

\author{
Vladimir Djoković, Dušan Kostoski, Miroslav D. Dramićanin, \\ and Edin Suljovrujić
}

Vinča Institute of Nuclear Science, P.O. Box 522, 11001 Belgrade, Yugoslavia

(Received February 8, 1999)

\begin{abstract}
The stress relaxation of unoriented $(\lambda=1)$ and oriented $(\lambda=9)$ high density polyethylene (HDPE) was studied for different levels of radiation $(0,100,300$, and $500 \mathrm{kGy})$. The relaxation behavior is analyzed in terms of a formerly established two-process model. In that model, one process is related to the crystal and the other to the amorphous fraction of a polyethylene sample. The modified viscoelastic properties of HDPE, due to effects of orientation and irradiation, are reflected in changes in viscosity coefficients and elasticity moduli of these two fractions. This made possible better comprehension of the nature of changes in the relaxation behavior of HDPE induced by irradiation and orientation.

KEY WORDS Stress Relaxation / Crystal Fraction / Amorphous Fraction / Polyethylene /
\end{abstract}

The effects of ionizing radiation and orientation on the mechanical relaxation of polyethylene have been the subject of recent publications. ${ }^{1-6}$ Some of these studies have shown an increase in intensity of the stress relaxation with orientation. ${ }^{1-3}$ This behavior is a consequence of the reduced mobility of highly stretched tie molecules in oriented samples. It has also been shown that radiation crosslinking can improve stability to applied stress up to $30 \% .^{2-6}$ Formation of network suppresses the cold flow and increases internal resistance of the sample.

For better understanding of the influence of irradiation and orientation on the relaxation behavior, in our previous paper ${ }^{4}$ we proposed a two-process model for the stress relaxation in linear low density polyethylene (LLDPE). The model is based on the assumption that the stress relaxation can be represented with two thermally activated processes acting in parallel, each related with one fraction of polyethylene sample: crystal or amorphous. The process which takes place in a crystal domain is, in fact, a propagation of defects through the lattice, as it has been established earlier by Wilding and Ward. ${ }^{7}$

This approach is explored further in the present paper by examining the effects of orientation and different adsorbed doses of radiation on the relaxation behavior of high density polyethylene (HDPE). Since the relaxation process is very sensitive to prior structure of the samples, we used differential scanning calorimetry (DSC) and gel measurements to find out the changes in the crystal fraction and the degree of network formation respectively.

\section{EXPERIMENTAL}

In these investigations HDPE HIPLEX EHM 6003 $\rho=0.96 \mathrm{~g} \mathrm{~cm}^{-3}$ and $M_{w}=76700$ was used. Preparation and irradiation of the samples are described in previous studies. $^{3-5}$ There are two types of samples, unoriented $(\lambda=1)$ and oriented $(\lambda=9)$, which were exposed to ${ }^{60} \mathrm{Co}$ gamma-source up to absorbed doses of $0,100,300$, and $500 \mathrm{kGy}$. Initial elasticity moduli of unirradiated samples were $E=0.6 \mathrm{GPa}$ for $\lambda=1$ and $E=2.9 \mathrm{GPa}$ for $\lambda=9$. These values were obtained from stress-strain measurements using Zwick tensile testing machine at a load rate of $20 \mathrm{~cm} \mathrm{~min}^{-1}$.

The stress relaxation measurements are also performed on a Zwick tensile machine using same load rate. Specimens were held at constant strain $(\varepsilon=3 \%)$ and constant temperature $\left(24^{\circ} \mathrm{C}\right)$ between 8 and $18 \mathrm{~h}$, decreasing of stress being continually monitored. Extraction in boiling xylene for $24 \mathrm{~h}$ was used for determination of the gel content. The melting endotherms of unconstrained samples were recorded on a Perkin Elmer DSC-2 at $10 \mathrm{~K} \mathrm{~min}^{-1}$ from 320 to $430 \mathrm{~K}$. The heat of fusion of each sample is determined from the area under the endotherm curve and the degree of crystallinity is calculated assuming a value of $289 \mathrm{~J} \mathrm{~g}^{-1}$ for $\Delta H_{\mathrm{f}}$ of a theoretical $100 \%$ crystalline polyethylene.

\section{RESULTS AND DISCUSSION}

Figures 1 and 2 depict the stress relaxation of unoriented $(\lambda=1)$ and oriented $(\lambda=9)$ samples for various irradiation doses respectively. In these figures the stress relaxation curves are normalized with respect to the initial stress $\sigma_{0}$ (stress at $t=0$ ). For both $\lambda=1$ and $\lambda=9$ residual stress increases with increasing irradiation dose, which shows a decrease in intensity of the stress relaxation. This is a consequence of the reduced cold flow due to crosslinking effects. ${ }^{2-6}$ It can also be seen that the shape of the relaxation curves is changed after orientation, but only slightly altered after irradiation for one specific draw ratio. A comparison between unirradiated samples of different draw ratios is given in the inset of Figure 1. Residual stress for $\lambda=1$ is larger than that for $\lambda=9$, suggesting better stability upon loading of unoriented samples, as already mentioned in the introduction.

Normally, the relaxation curves consist of two parts: exponential and power-law region. ${ }^{8}$ In the beginning of the relaxation process, stress decays rapidly (exponential part) and after that passes into slow decay (power law). For the unoriented sample, duration of exponential decay is approximately $100 \mathrm{~s}$, while for the oriented sample it is only $0.5 \mathrm{~s}$. Such behavior of the oriented HDPE is in conflict with our previous results 


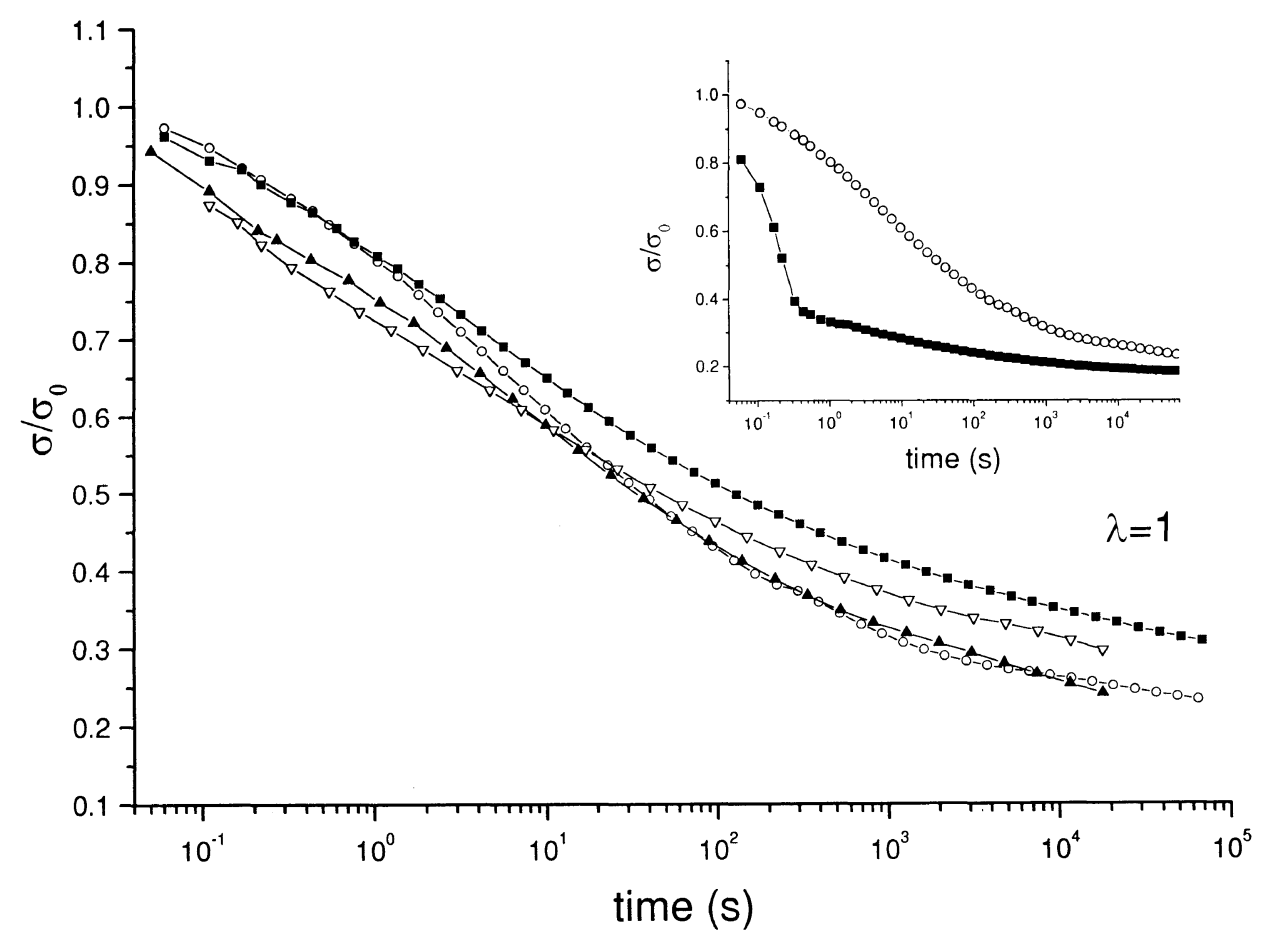

Figure 1. Stress relaxation curves of unoriented $(\lambda=1)$ HDPE normalized with respect to initial stress $\left(\sigma_{0}\right)$ for various levels of radiation: 0 $(\bigcirc), 100(\boldsymbol{\Delta}), 300(\nabla)$, and $500(\boldsymbol{\square}) \mathrm{kGy}$. Inset shows normalized relaxation curves of unirradiated $\lambda=1(\mathrm{O})$ and $\lambda=9(\boldsymbol{\square})$ samples.

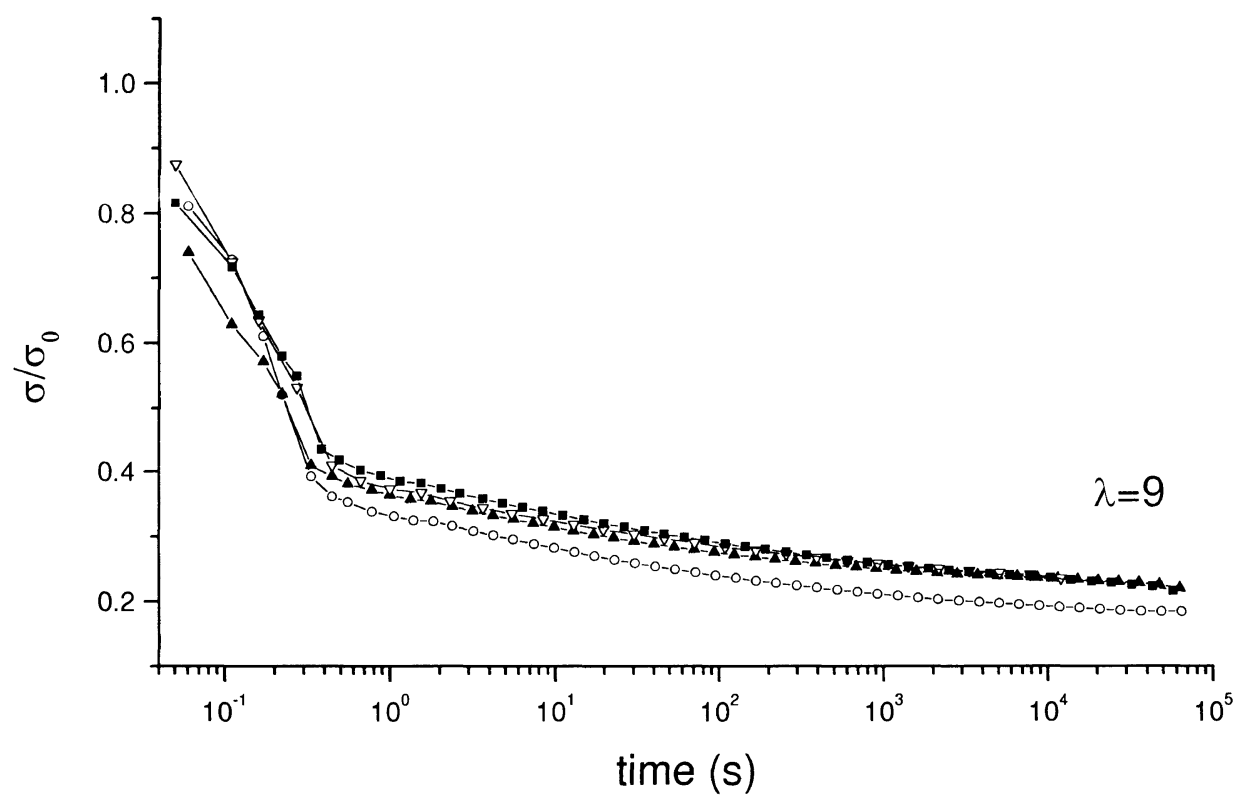

Figure 2. Stress relaxation curves of oriented $(\lambda=9)$ HDPE normalized with respect to initial stress $\left(\sigma_{0}\right)$ for various levels of radiation: $0(O)$, $100(\mathbf{\Delta}), 300(\nabla)$, and $500(\boldsymbol{\square}) \mathrm{kGy}$.

for the oriented ultra-high molecular weight polyethylene (UHMWPE) $^{5}$ and the LLDPE. ${ }^{3}$ Obviously, formation of fibrous structure due to stretching significantly influences the relaxation processes in HDPE. It should be emphasized that $\lambda=9$ is the maximal draw ratio we could obtain with this simple orientation technique.

In order to explain the relaxation behavior of HDPE, as in previous papers, ${ }^{4,5}$ we assume that a satisfactory representation may be given by the superposition of two thermally activated processes acting in parallel, using time-dependent viscosity coefficients

$$
\eta_{i}=\eta_{i}^{o}\left(1+\frac{t}{\tau_{i}}\right)
$$

assigning $i=1,2$ to each process. The two-process model is continuum-mechanical model which is shown in Figure 3. Decaying of stress in this system is given by the following equation

$$
\sigma(t)=\varepsilon E_{1}\left(1+\frac{t}{\tau_{1}}\right)^{-\left(E_{1} / \eta_{1}^{0}\right) \tau_{1}}+\varepsilon E_{2}\left(1+\frac{t}{\tau_{2}}\right)^{\left(E_{2} / \eta_{1}^{0}\right) \tau_{2}},
$$

where $\sigma$ is the stress, $\varepsilon$ is the strain, $t$ is the time, $E_{1}, E_{2}$ 
the Young moduli, and $\eta_{1}, \eta_{2}$ the viscosity coefficients. In the fitting procedure, by using eq 2 it is possible to find coefficients $\eta_{1}^{0}$ (from now on $\eta_{1}$ ), $\eta_{2}^{0}\left(\eta_{2}\right)$, and moduli $E_{1}, E_{2}$ of the crystal and amorphous fraction. We shall adopt $\tau_{1}=\tau_{2}=1$ 's in order to preserve full time-scale equality and consistency of evaluated viscoelastic parameters with Maxwell-Wichert model $^{9}$

$$
\sigma(t)=\varepsilon E_{1} \exp \left(-\frac{E_{1}}{\eta_{1}} t\right)+\varepsilon E_{2} \exp \left(-\frac{E_{2}}{\eta_{2}} t\right) .
$$

Former model (valid at times less than few seconds) is based on the same multielement combination (Figure 3) as our model, but assumes time independent viscosity coefficients.

Fits to eq 2 of the original experimental curves are given by the solid line in Figures 4 and 5 . In the fitting

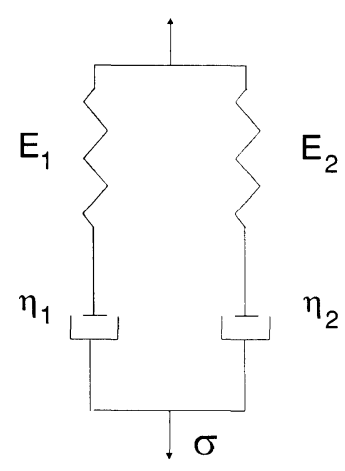

Figure 3. Two Maxwell elements (dashpot and spring in the line) connected in parallel as representation of two-process model. Each Maxwell element represents one fraction of polyethylene.

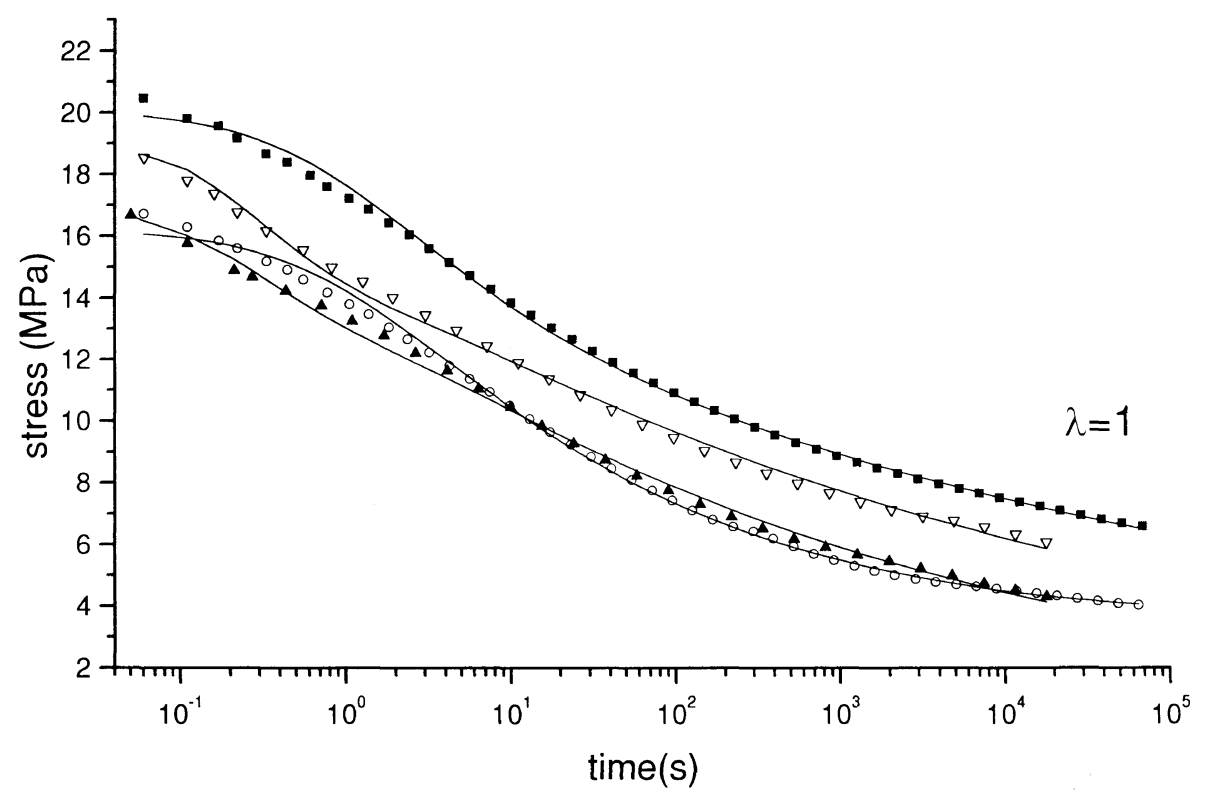

Figure 4. Decaying of stress versus time of unoriented $(\lambda=1)$ HDPE, unirradiated $0(\bigcirc)$, and irradiated with $100(\mathbf{\Delta}), 300(\nabla)$, and $500(\mathbf{B}$ kGy in air. Fit to eq 2 is given with solid line.

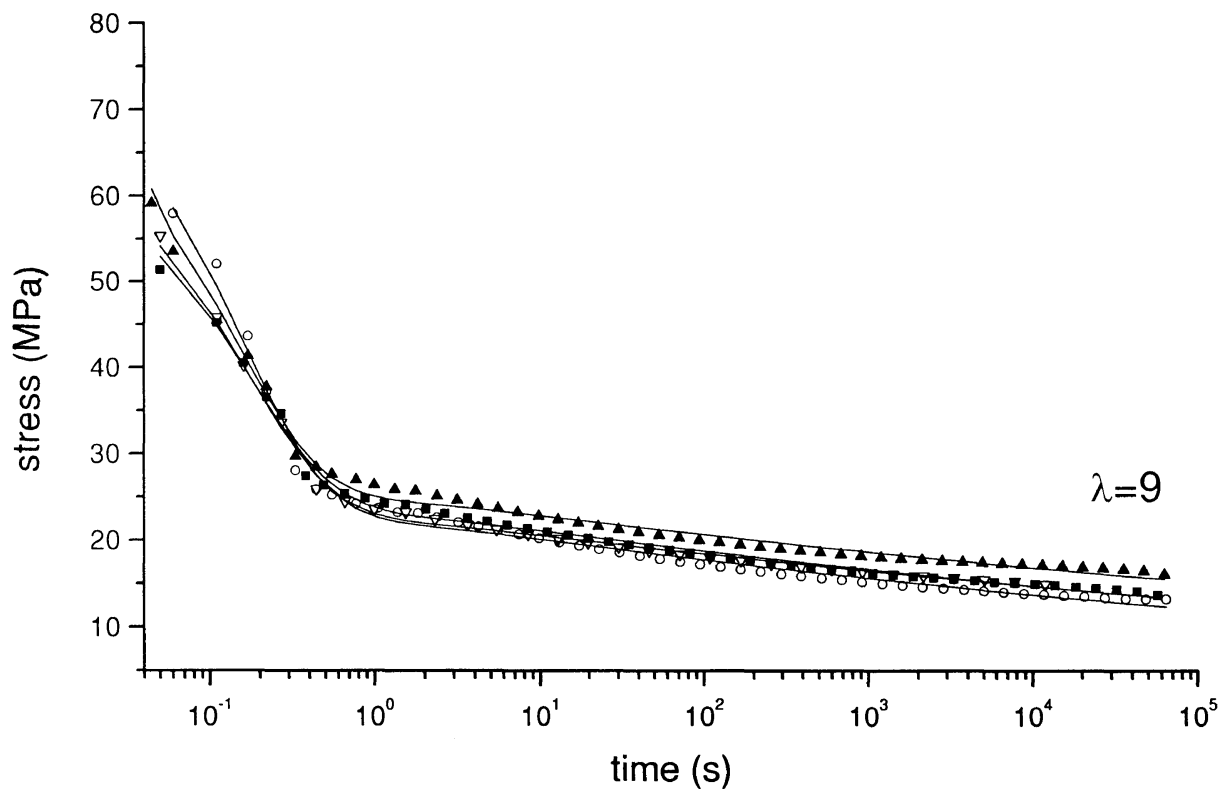

Figure 5. Decaying of stress versus time of oriented $(\lambda=9)$ HDPE, unirradiated $0(O)$, and irradiated with $100(\mathbf{\Delta}), 300(\nabla)$, and 500 (ם) $\mathrm{kGy}$ in air. Fit to eq 2 is given with solid line. 


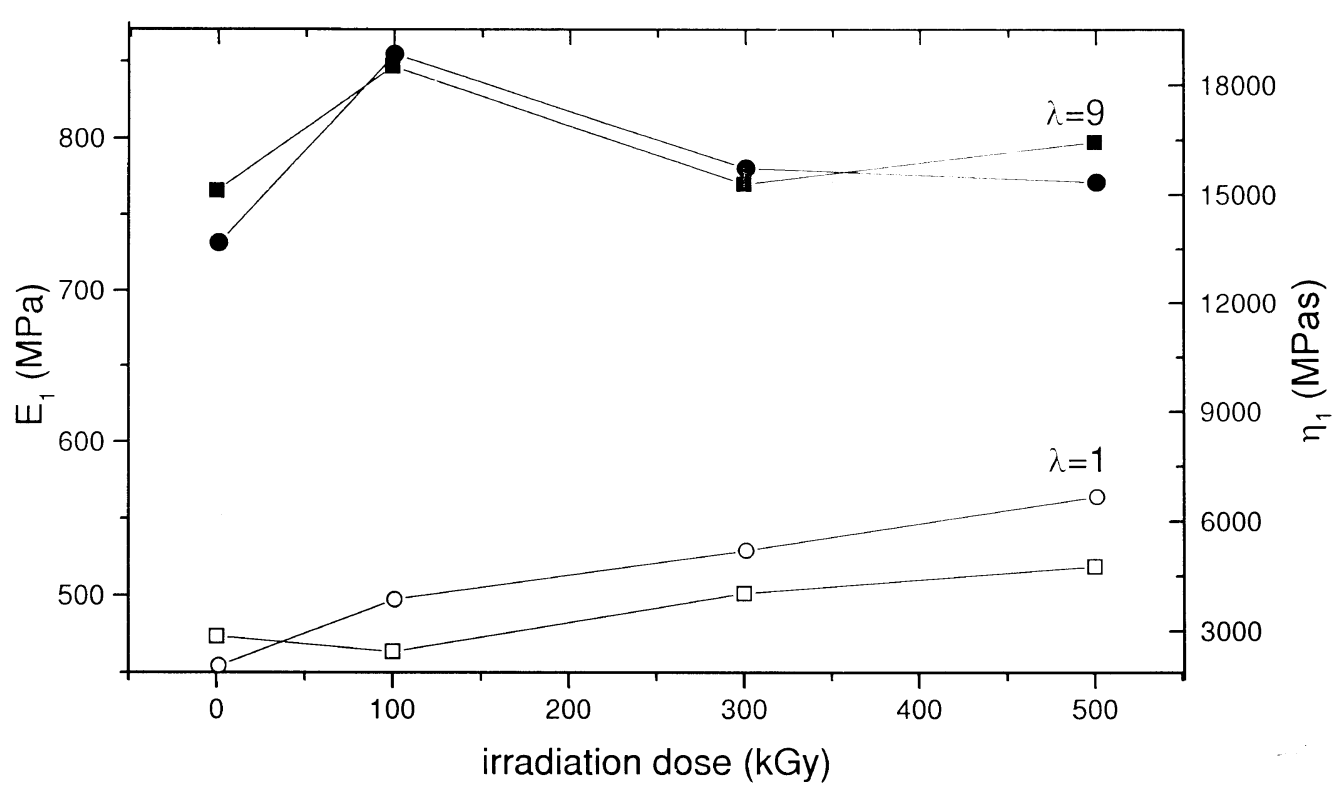

Figure 6. Coefficient of viscosity $\eta_{1}$ (circles) and Young moduli $E_{1}$ (squares) of crystal fraction for two different draw ratios: $\lambda=1$ (open symbols) and $\lambda=9$ (full symbols).

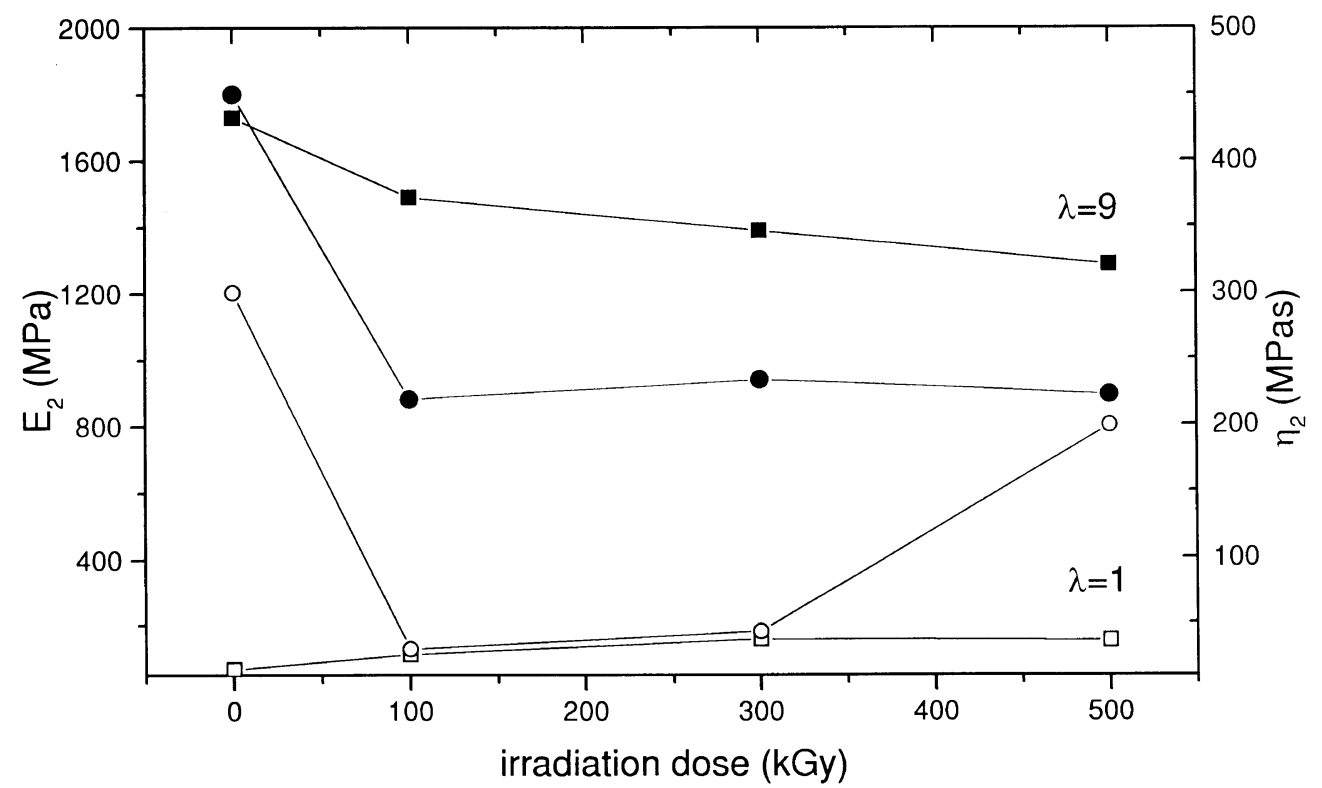

Figure 7. Coefficient of viscosity $\eta_{2}$ (circles) and Young moduli $E_{2}$ (squares) of amorphous fraction for two different draw ratios: $\lambda=1$ (open symbols) and $\lambda=9$ (full symbols).

procedure we used Hooke-Jeeves non-linear estimation method. Figures 6 and 7 show the obtained coefficients and moduli plotted versus irradiation dose. Assuming that the relaxation process associated with the crystal fraction is a consequence of the $c$-axis slip in crystal lamellae $^{6}$ or a propagation of the defects in the lattice, ${ }^{7}$ it can be concluded that such process must be characterized by a larger value of viscosity coefficient (or a smaller activation volume ${ }^{4}$ ) than the coefficient associated with the amorphous fraction. Because of that, process 1 is associated with the crystal and process 2 with the amorphous fraction.

Table I shows that the gel content of the $\lambda=1$ sample even at $100 \mathrm{kGy}$ reaches $50 \%$. It has been shown ${ }^{10,11}$ that radiation induced crosslinking occurs preferentially on the surfaces of lamellae. Therefore, the crystal viscosity $\eta_{1}$ for the $\lambda=1$ sample will increase as

Polym. J., Vol. 31, No. 12, 1999
Table I. Gel content as a function of irradiation dose

\begin{tabular}{|c|c|c|c|}
\hline \multicolumn{2}{|c|}{$\lambda=1$} & \multicolumn{2}{|c|}{$\lambda=9$} \\
\hline Irradiation dose & \multirow{2}{*}{ Gel content $/ \%$} & Irradiation dos & \\
\hline kGy & & kGy & \\
\hline 100 & 50 & 100 & 47 \\
\hline 300 & 73 & 300 & 70 \\
\hline 500 & 74 & 500 & 73 \\
\hline
\end{tabular}

irradiation dose increases due to "shield" effects ${ }^{4}$ of the surface network (Figure 6). This is in agreement with our results for unoriented and oriented LLDPE and UHMWPE ${ }^{4,5}$ and also with decreasing of activation volume observed in creep measurements. ${ }^{12}$ Table II shows the heats of fusion, melting temperatures and 


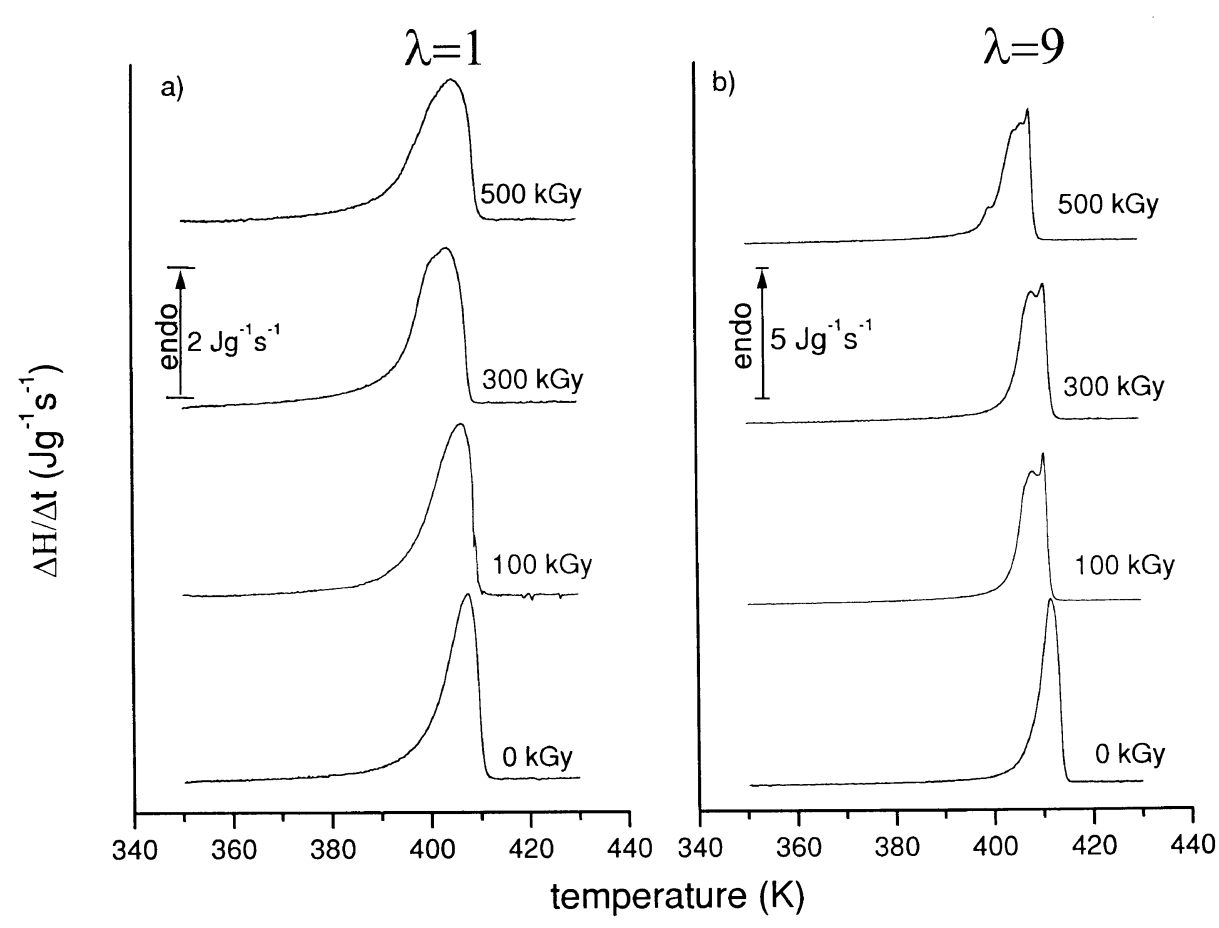

Figure 8. Melting endotherms of a) unoriented and b) oriented HDPE for different irradiation doses.

crystallinity which are related to melting endotherms of unoriented samples in Figure 8a. Irradiation produces an increase in the heat of fusion and consequently in crystallinity due to scission of tie molecules. ${ }^{13}$ This process, together with crosslinking effects at the surface of lamellae, leads to an increase ${ }^{4}$ of crystal modulus $E_{1}$ of the $\lambda=1$ sample as dose increases (Figure 6), although at $500 \mathrm{kGy}$ dose, due to a great number of defects, crystallinity drops.

Transformation from spherulit to fibrous structure due to the orientation process induces better perfection of crystalline regions and higher degree of crystallinity with respect to the initial material (Figures $8 \mathrm{a}$ and $8 \mathrm{~b}$ ) and Table II). Wilding and Ward ${ }^{14}$ have shown that the activated event becomes more localized if the structure has higher degree of molecular alignment and perfection. Since the activation event, through activation volume, is directly related to viscosity coefficient, larger values of crystal viscosity $\eta_{1}$ will be obtained due to the orientation process (Figure 6). Also, as a consequence of the increase in crystallinity and continuity ${ }^{14}$ of crystal fraction, modulus $E_{1}$ of the oriented sample will be larger than that of the unoriented one (Figure 6).

Due to specific structure of oriented samples, $E_{1}$ and $\eta_{1}$ do not show the same dependence with increasing irradiation dose as unoriented samples. Fibrous structure of the oriented samples possesses a large number of taut-tie molecules which can be considered as defects in crystal structure at their points of entry to the lattice. ${ }^{15}$ Chain scission effects will produce relief of strain regions of the crystal, ${ }^{13,15}$ hence, an increase of $E_{1}$ and $\eta_{1}$ for $100 \mathrm{kGy}$ dose is the consequence of increasing crystallinity and perfection of crystalline domains (Figure 6). This effect can also be related to the highest initial stress of the oriented $100 \mathrm{kGy}$ irradiated sample (Figure 5) in contrast to unoriented samples where the initial stress increases steadily with irradiation. Since process 1 is 1198
Table II. Effects of irradiation dose on heats of fusion $\left(\Delta H_{\mathrm{f}}\right)$, melting temperatures $\left(T_{\mathrm{m}}\right)$, and crystallinity $(\chi)$ of unoriented and oriented HDPE

\begin{tabular}{|c|c|c|c|c|c|c|c|}
\hline & $\lambda=1$ & & & & $\lambda=9$ & & \\
\hline \multirow{2}{*}{$\begin{array}{c}\text { Irradiation } \\
\text { dose } / \mathrm{kGy}\end{array}$} & \multicolumn{3}{|c|}{$\Delta H_{\mathrm{f}} \pm 0.2$} & \multirow{2}{*}{$\begin{array}{c}\text { Irradiation } \\
\text { dose/kGy }\end{array}$} & \multirow{2}{*}{$\frac{\Delta H_{\mathrm{f}} \pm 1}{\mathrm{Jg}^{-1}}$} & \multirow{2}{*}{$T_{\mathrm{m}} / \mathrm{K}$} & \multirow{2}{*}{$\chi / \%$} \\
\hline & $\mathrm{J} \mathrm{g}^{-1}$ & & & & & & \\
\hline 0 & 159.2 & 407 & 55.1 & 0 & 202 & 411 & 69.9 \\
\hline 100 & 173.4 & 406 & 60.0 & 100 & 206 & 410 & 71.3 \\
\hline 300 & 195.6 & 403 & 67.7 & 300 & 199 & 410 & 68.8 \\
\hline 500 & 180.3 & 404 & 62.4 & 500 & 192 & 407 & 66.4 \\
\hline
\end{tabular}

connected with the beginning of stress relaxation, ${ }^{4,6}$ lower values of initial stress means lower perfection and elasticity of crystalline domains. Therefore, lower values of $E_{1}$ and $\eta_{1}$ are obtained in oriented samples for 300 and $500 \mathrm{kGy}$ doses (Figure 6). The former statement can be argued with respect to the formation of new thin lamellae manifested as low temperature peak on the melting endotherm of oriented samples (Figure $8 \mathrm{~b}$ ). The obtained new lamellae are not perfect as those that have already existed, which is probably the reason why at higher doses $(>100 \mathrm{kGy})$ due to large number of defects, $\eta_{1}$ and $E_{1}$ decrease despite the high gel content (Figure 6).

High concentration of extremely strained molecules in the amorphous fraction, obtained by orientation, induces characteristic properties which are reflected in differences between $E_{2}$ and $\eta_{2}$ for these two types of samples (Figure 7). By comparing modulus $E_{2}$ for $\lambda=9$ (approximately $1.75 \mathrm{GPa})$ with the same modulus for $\lambda=1(60 \mathrm{MPa}) \mathrm{a}$ well known conclusion can be drawn that the orientation process significantly enhances elasticity of polyethylene along the direction of stretching. On the other hand, conformation mobility of highly strained tie molecules 
is limited. Therefore, cold flow in oriented HDPE will be more pronounced than in unoriented, giving larger values of $\eta_{2}$ (Figure 7). Amorphous viscosity $\eta_{2}$ of both samples decreases after irradiation due to crosslinking effects (Table I) which prevent cold flow (Figure 7). The apparent growth of $\eta_{2}$ at $500 \mathrm{kGy}$ for the $\lambda=1$ sample is the consequence of detrimental effects of irradiation at high doses, which is in agreement with our previous paper. ${ }^{4}$ Namely, gel content tends to saturation at $300 \mathrm{kGy}^{2,16}$; therefore at higher doses irradiation produces defects in the network which enhance cold flow. This effect is not evident in the $\lambda=9$ samples probably because of the lowering of amorphous fraction in the orientation process. Modulus $E_{1}$ of unoriented HDPE is only slightly changed with irradiation suggesting that the improvement of stability to loading is rather the consequence of the inhibition of cold flow due to crosslinking than of the enhancement in elasticity of the network. Large number of constrain molecules in amorphous fraction of oriented HDPE, which are responsible for high elasticity, are at the same time more liable to chain scission. Presumably, these effects induce the decrease in $E_{2}$ along the draw direction with increasing irradiation dose despite the crosslinking effects which suppress slippage of strained molecules (Figure 7).

It should be noted, that opposite to unoriented samples, modulus of elasticity of amorphous fraction $E_{2}$ for oriented samples has a larger value than the modulus of crystal fraction $E_{1}$ (Figures 6 and 7). This is in conflict with our previous studies ${ }^{4,5}$ and a possible reason for the rapid stress drop (Figures 2 and 5). As already mentioned, highly strained molecules can be considered as defects in crystal structure at their points of entry to the lattice. During fast loading $\left(20 \mathrm{~cm} \mathrm{~min}^{-1}\right)$ of oriented specimen, due to stress concentration at the defects points, it seems easier for the molecules in the crystal to be pulled out from the lattice than for the constrained molecules to slide by each other. After a short period of time, due to relief of strain at the defects, stress is distributed on taut-tie molecules and passes into slow decay.

It has been shown by X-ray measurements that the modulus of polyethylene crystal along the chain axis is $240-300 \mathrm{GPa}{ }^{17,18}$ Estimated values of modulus $E_{1}$ $(450-850 \mathrm{MPa})$ are much lower due to influence of stress concentration. ${ }^{19}$ Very high rate of loading is also the reason for the low values of crystal modulus $E_{1}$, because the structure is unable to adjust to applied stress. Similar trend is observed by using high stress amplitudes in dynamical measurements. ${ }^{20}$

\section{CONCLUSIONS}

Presented results have shown that orientation, as well as irradiation, significantly affects the stress relaxation behavior of HDPE. As in previous studies ${ }^{4,5}$ the stress relaxation is described by two thermally activated processes acting in parallel. The first, characterized by a high coefficient of viscosity $\left(\eta_{1}\right)$, is associated to crystal fraction. The second process is characterized by a small viscosity coefficient $\left(\eta_{2}\right)$ and corresponds to the amorphous fraction. Changes in elasticity moduli and viscosity coefficients of both fractions due to irradiation of a $\lambda=1$ sample have revealed similar behavior as in investigations on the other types of polyethylene (LLDPE and UHMWPE). ${ }^{4,5}$ Contrary to our previous results, orientation leads to drastic changes in the relaxation properties. Exponential region of the relaxation curve is reduced to approximately $0.5 \mathrm{~s}$ compared to $100 \mathrm{~s}$ for unoriented HDPE. Obviously, a large number of taut-tie molecules with limited conformational mobility and a high degree of crystallinity $(\chi>70 \%)$ in oriented HDPE are the reason for this behavior. This is reflected in larger values of amorphous $E_{2}$ than of crystal $E_{1}$ moduli of oriented samples. Nevertheless, irradiation will improve stability to applying load for both samples $(\lambda=1$ and $\lambda=9$ ), because crosslinking effects will reduce the cold flow and enhance internal resistance of the structure.

This study also confirmed, that establishing of the dependence of characteristic coefficients and moduli of the amorphous and crystal fraction upon irradiation and orientation offers good insight into the influence of these effects on the relaxation behavior of HDPE.

\section{REFERENCES}

1. Y. M. Boiko, W. Brostow, A. Y. Goldman, and A. C. Ramamurthy, Polymer, 36, 1383 (1995).

2. Y. M. Boiko and V. V. Kovriga, Int. J. Polym. Mater., 22, 209 (1993).

3. V. Djoković, Z. Kačarević-Popović, D. Kostoski, and D. Dudić, Polym. Degrad. Stab., 61, 73 (1998).

4. V. Djoković, D. Kostoski, S. Galović, M. D. Dramićanin, and Z. Kačarević-Popović, Polymer, 40, 2631 (1999).

5. V. Djoković, D. Kostoski, M. D. Dramićanin, and D. Dudić, Rad. Phys. Chem., 55, 605 (1999).

6. S. K. Bhateja and E. H. Andrews, J. Appl. Polym. Sci., 34, 2809 (1987).

7. M. A. Wilding and I. M. Ward, J. Mater. Sci., 19, 629 (1984).

8. Ch. Högfors, J. Kubát, and M. Rigdahl, Phys. Stat. Sol., 107, 147 (1981).

9. J. R. Fried, "Polymer Science and Technology," Prentice Hall, Englewood Cliffs, N.J., 1995, pp 196-205.

10. G. Ungar, J. Mater. Sci., 16, 2635 (1981).

11. R. Salovey, D. C. Basset, J. Appl. Phys., 35, 3216 (1964)

12. P. G. Klein, D. W. Woods, and I. M. Ward, J. Polym. Sci. Polym. Phys., 25, 1359 (1987).

13. Z. Kačarević-Popović, D. Kostoski, Z. Stojanović, and V. Djoković, Polym Degrad. Stab., 56, 227 (1997).

14. I. M. Ward and M. A. Wilding, J. Polym. Sci. Polym. Phys., 22, 561 (1984).

15. S. K. Bhateja, S. M. Yarbrough, and E. H. Andrews, J. Macromol. Sci. Phys., B29, 1 (1990).

16. G. Akay, F. Cimen, and T. Tincer, Rad. Phys. Chem., 36, 337 (1990).

17. I. Sakurada, T. Ito, and K. Nakamae, J. Polym. Sci., Part C, 15, 75 (1966).

18. J. Clements, R. Jakeways, and I. M. Ward, Polymer, 19, 639 (1978).

19. H. Tianbai, Polymer, 27, 253 (1986)

20. J. D. Ferry, "Viscoelastic Properties of Polymers," John Wiley \& Sons, New York, N.Y., 1961, pp 349 